\title{
Higher harmonic generation microscopy for developmental biology
}

\author{
Chi-Kuang Sun, ${ }^{\text {a,* }}$ Shi-Wei Chu, ${ }^{\text {a }}$ Szu-Yu Chen, ${ }^{\text {a }}$ Tsung-Han Tsai, ${ }^{\text {a }}$ Tzu-Ming Liu, ${ }^{\text {a }}$ \\ Chung-Yung Lin, ${ }^{b}$ and Huai-Jen Tsai ${ }^{b}$ \\ ${ }^{a}$ Graduate Institute of Electro-Optical Engineering and Department of Electrical Engineering, National Taiwan University, Taipei 10617, Taiwan, ROC \\ ${ }^{\mathrm{b}}$ Institute of Molecular and Cell Biology, National Taiwan University, Taipei 10617, Taiwan, ROC
}

Received 8 July 2003, and in revised form 6 October 2003

\begin{abstract}
Optical higher harmonic generation, including second harmonic generation and third harmonic generation, leaves no energy deposition to its interacted matters due to an energy-conservation characteristic, providing the "noninvasiveness" nature desirable for biological studies. Combined with its nonlinearity, higher harmonic generation microscopy provides excellent three-dimensional (3D) sectioning capability, offering new insights into the studies of embryonic morphological changes and complex developmental processes. By choosing a laser working in the biological penetration window, here we present a noninvasive in vivo light microscopy with sub-micron 3D resolution and millimeter penetration, utilizing endogenous higher harmonic generation signals in live specimens. Noninvasive imaging was performed in live zebrafish (Danio rerio) embryos. The complex developmental processes within $>1$ mm-thick zebrafish embryos can be observed in vivo without any treatment. No optical damage was found even with high illumination after long-term observations and the examined embryos all developed normally at least to the larval stage. The excellent $3 \mathrm{D}$ resolution of the demonstrated technology allows us to capture the subtle developmental information on the cellular or subcellular levels occurring deep inside the live embryos and larvae. This technique can not only provide in vivo observation of the cytoarchitecture dynamics during embryogenesis with submicron resolution and millimeter penetration depth, but would also make strong impact in developmental and structural biology studies.
\end{abstract}

(c) 2003 Elsevier Inc. All rights reserved.

Keywords: Higher harmonic generation; Developmental biology; Embryo; Multi-dimensional microscopy; Scanning microscopy; Second harmonic generation; Third harmonic generation

\section{Introduction}

To understand the complex developmental processes in vertebrates, a noninvasive means to visualize the subcellular changes is necessary. Imaging modalities such as computed tomography (CT) (Battaglia, 2003), magnetic resonance imaging (MRI) (Greicius, 2003), ultrasound imaging, are examples of well-developed technologies for visualizing internal biological structures in vivo. The costly instruments and slow acquisition speed of CT and MRI post limitation on in vivo developmental dynamic studies and none of them are capable of visualizing the cellular and subcelluar structures smaller than $10-\mu \mathrm{m}$. Ultrasound possesses the potential to image deeply into the biological specimens,

\footnotetext{
${ }^{*}$ Corresponding author. Fax: +886-2-2367-7467.

E-mail address: sun@cc.ee.ntu.edu.tw (C.-K. Sun).
}

but its resolution is limited to $\sim 50-\mu \mathrm{m}$ even with high acoustic frequencies $(40-100 \mathrm{MHz})$ (Turnbull et al., 1995). Recently, several optical techniques have been applied to the field of developmental biology, e.g., optical coherence tomography (OCT) (Boppart et al., 1997), confocal laser scanning microscopy (CLSM) (Palmes-Saloma and Saloma, 2000), and two-photon fluorescence microscopy (2PFM) (Phillips et al., 2001). OCT, which is analogous to ultrasound imaging except that it uses optical waves instead of acoustic waves, has demonstrated $\sim 1-\mu \mathrm{m}$ axial resolution (Drexler et al., 2001; Povazay et al., 2002) with $\sim 10-\mu$ m lateral resolution. This resolving power is good enough for cellular imaging but still inferior to that of nonlinear microscopies including multi-photon fluorescence microscopy and higher harmonic generation microscopy. The out-of-focus image degradation problem of OCT also puts an impediment to its application to developmental 
biology studies. Confocal laser scanning microscopy provides a significant improvement in axial and lateral resolutions over conventional epifluorescence microscopy by eliminating out-of-focus fluorescence with a spatial filter in the form of a confocal aperture. However, due to the high attenuation of visible/ultraviolet excitation light, the single-photon based confocal microscopy is not able to provide high penetration capability. Its out-of-focus absorption would not only cause out-of-focus photo bleaching but also cell damages (Denk et al., 1990; Stelzer et al., 1994). Utilizing twophoton induced fluorescence with near-infrared (NIR) excitation, Denk et al. (1990) demonstrated 2PFM. With a quadratic dependence on the laser intensity and the NIR wavelength, 2PFM showed high axial/depth discrimination even without a confocal pinhole. The outof-focus absorption is strongly reduced due to the nonlinear nature, along with the reduction of out-offocus photobleaching and photodamage. However, this microscopic technique still requires on-focus absorption and high optical illumination to stimulate nonlinear fluorescence for imaging purpose. This high on-focus absorption, which may induce photodamage/photobleach effects, and the general needs of exogenous fluorophores make this technique not truly 'noninvasive.'

In contrast to laser-induced fluorescence higher harmonic generation (HHG), including second harmonic generation (SHG), and third harmonic generation (THG), is known to leave no energy deposition to its interacted matters due to the energy conservation characteristic and the emitted HHG photon energy is the same as the annihilated excitation photon energy. This energy-conservation characteristic releases no energy to its interacted specimens and provides the optical "noninvasive" nature desirable for microscopy applications, especially for clinical imaging (Brown et al., 2003; Chu et al., 2001; Guo et al., 1997; Peleg et al., 1999). Due to its nonlinear nature, the generated SHG intensity depends on square of the incident light intensity, while the generated THG intensity depends on cubic of the incident light intensity. Similar to multiphoton induced fluorescence processes, these nonlinear dependencies allow localized excitation and provide intrinsic sectioning capability. It is thus important to utilize these endogenous HHG signals (that is sometimes stronger than multi-photon induced autofluorescence) for imaging purpose to replace unnecessary usage of invasive and toxic fluorophores in common multi-photon microscopy. Here we present an in vivo vertebrate developmental biology study using our developed HHG microscopy based on a femtosecond Cr:forsterite laser, whose wavelength falling in the transparency window of most biological tissues and thus allowing maximized optical penetration as well as minimized photodamages (Chen et al., 2002). The in vivo study is performed on live free-moving zebrafish (Danio rerio) embryos and larvae without any treatment. By using the endogenous HHG sources as the microscopic contrast agents, no fluorescence is required and the common issues of photodamage, phototoxicity, photobleaching, dye availability, or dye toxicity can all be eliminated and therefore this technique can be regarded as a truly "noninvasive" imaging modality. Complex developmental processes throughout the 1.5 -mm-thick zebrafish embryos from initial cell proliferation, gastrulation, to the tissue formation can all be continuously visualized in vivo without any treatment on the live specimens. No optical damage can be found after long-term (12-h) continuous observation with $100-\mathrm{mW}$ average incident power on one embryo, corresponding a total exposure of over $1000 \mathrm{~J}$, and the examined embryos can all develop normally at least to the larval stage, confirming the noninvasive nature of the developed technology. The excellent 3D resolution (sub-micron) of the demonstrated microscopy and its high penetration $(1.5 \mathrm{~mm}$ into the surface of the live specimen) allow us to continuously capture the subtle developmental information on the cellular or sub-cellular levels occurred deep inside the live embryos and larva.

\section{Nature of higher harmonic generation}

SHG and THG are both nonlinear processes, related to the interaction of intense light with matters. SHG describes the generation of light waves that are twice the frequency (with half of the original wavelength) of the original waves while THG describes the generation of light waves that triple the frequency (with one-third of the original wavelength) of the original waves. The higher harmonic light wave generation is coupled from the excited nonlinear polarization $P^{\mathrm{NL}}$ under intense laser excitation. The relationship between the induced nonlinear polarization $P^{\mathrm{NL}}$ and the excitation light is usually described through a nonlinear susceptibility $\chi$. For example, SHG can be generated through a secondorder nonlinear polarization created according to

$P^{\mathrm{NL}}(2 \omega)=\frac{1}{2} \varepsilon_{0} \chi^{(2)}(2 \omega: \omega, \omega) E(\omega) E(\omega)$,

where $\chi^{(2)}(2 \omega: \omega, \omega)$ is the second-order nonlinear susceptibility responsible for SHG and $E(\omega)$ is the laser field amplitude. $P^{\mathrm{NL}}(2 \omega)$ is the induced SHG polarization, which in turn generates light field radiation $E(2 \omega)$ at twice the original angular frequency $\omega$. Similarly, THG is related to the creation of a third-order nonlinear polarization (at $3 \omega$ angular frequency) according to

$P^{\mathrm{NL}}(3 \omega)=\frac{1}{4} \varepsilon_{0} \chi^{(3)}(3 \omega: \omega, \omega, \omega) E(\omega) E(\omega) E(\omega)$,

where $\chi^{(3)}(3 \omega: \omega, \omega, \omega)$ represents the third-order nonlinear susceptibility responsible for THG. 
SHG and THG can be visualized by considering the interaction in terms of exchange of photons between various frequencies of the fields. According to this picture, which is illustrated in Fig. 1, two or three photons of angular frequency $\omega$ are destroyed and a photon of angular frequency $2 \omega$ (for SHG) or $3 \omega$ (for THG) is simultaneously created in a single quantum-mechanical process. The solid lines in the figure represent the atomic ground states, and the dashed lines represent what are known as virtual levels. These virtual levels are not energy eigenlevels of the atoms, but rather represent the combined energy of one of the energy eigenstates of the atom and one or more photons of the radiation field. Due to its virtual level transition characteristic, HHG is known to leave no energy deposition to the interacted matters, since no real transition involves and the emitted photon energy is exactly the same as the total absorbed photon energy, also referring as the energy conservation law. This virtual-transition and energy-conservation characteristics provide the optical "noninvasive" nature desirable for microscopy applications, especially for live specimen imaging.

According to Eqs. (1) and (2), the generated SHG intensity depends on the square of the incident light intensity, while the generated THG intensity will depend on the cubic of the incident light intensity. Similar to 2PFM, SHG and THG microscopy (Gannaway and Sheppard, 1978) provides superior axial resolution due to quadratic and cubic dependencies of the signals on the illumination intensity. Usually the third-order nonlinear susceptibility $\chi^{(3)}(3 \omega: \omega, \omega, \omega)$ responsible for THG is much weaker than the second-order nonlinear susceptibility $\chi^{(2)}(2 \omega: \omega, \omega)$ responsible for SHG, thus THG is harder to observe. However, not all materials have second-order nonlinear susceptibility. For centrosymmetric media, the lowest order nonlinear susceptibility will be $\chi^{(3)}$ instead of $\chi^{(2)}$. Random distribution of biomolecules and suborganelles inside tissues and cells create optical centro-symmetry in the optical wavelength $(0.4-1-\mu \mathrm{m})$ scale, thus inhibiting SHG. SHG is only allowed in noncentro-symmetric media. On the other hand, all materials allow the creation of third-order susceptibility, of which the magnitudes vary according to material properties and wavelengths.
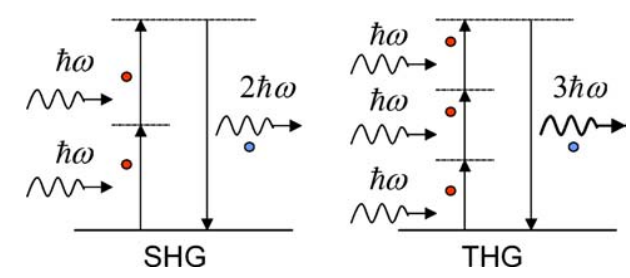

Fig. 1. Energy level diagrams describing (left) second-harmonic generation and (right) third-harmonic generation processes.
Since SHG does not occur in optically centro-symmetric media, SHG microscopy was first demonstrated for the studies of SHG photonic crystals (Gannaway and Sheppard, 1978), surfaces/interfaces (Shen, 1989), field distribution in semiconductors (Sun et al., 2000, 2001), and was then applied to biological studies including membrane potential imaging (Campagnola et al., 1999; Moreaux et al., 2000; Peleg et al., 1999) and tissue polarity examination (Freund et al., 1986; Guo et al., 1997). Our previous study has indicated that the contrast in sub-micron-resolution SHG images is created by organized nano-structures in biological samples where optical centro-symmetry is broken (Chu et al., 2002). Other studies also indicate the importance of structural proteins as the sources of SHG (Campagnola et al., 2002). On the other hand, due to the optical dispersion property in biological tissues, THG was proven to be generated from regions with optical inhomogeneity and was applied to image general cellular and sub-cellular structures (Barad et al., 1997; Chu et al., 2001; Müller et al., 1998). Here we use THG microscopy for general interface imaging, in comparison to SHG microscopy for nano-structures.

According to the momentum conservation law, the generated higher harmonic photons will be emitted in the same direction as the incident photons, unless processes like reflection, scattering, or noncollinear phase matching are involved. This forward emission property restricts the light collection geometry in most microscopy applications. While some SHG microscopic studies adopt the reflection geometry for its easy combination with current 2PFM setup (Yeh et al., 2002; Zoumi et al., 2002) or due to difficulties in the transmission collection (Brown et al., 2003), most SHG and THG microscopes utilize transmission detection nowadays.

\section{Excitation source selection}

To realize in vivo noninvasive HHG microscopy with high penetration and long-term observation capabilities, it is important to choose a correct laser source to avoid any unnecessary laser-tissue interactions. Since the generation of optical higher harmonics has a weak dependency on the excitation wavelength, we are thus allowed to choose the desirable excitation wavelength that has high penetration through turbid specimens with minimized unwanted light-tissue interactions including scattering, absorption, and photodamages (König et al., 1997). Due to the combination of diminishing scattering cross-section and avoiding resonant molecular absorption of common tissue constituents such as water, light attenuation in live biological specimen reaches a minimum around 1200-1350-nm wavelength region (Anderson and Parish, 1981; Bouma et al., 1996; Chu et al., 2001). We choose our excitation wavelength to be in the 
$1200-1350$-nm regime not only to provide high penetration and low photodamages but also to allow both SHG/THG within the visible spectrum.

Another important issue is the pulse energy and repetition rate selections. To maintain cell vitality, the optical peak intensity and the pulse energy should be lowered down to the safety region, which might limit the signal strength of the stimulated higher harmonic generation, thus limit the scanning speed and the image frame rate. Increasing the pulse repetition rate can solve this problem. Different from absorption-stimulated fluorescence, the virtual-transition process of higher harmonic generation does not create any real-transitionresulted upper-state electrons. The selection of the pulse repetition rate is thus not restricted by the relaxation time of those upper-state electrons. By increasing the repetition rate to higher than $100-\mathrm{MHz}$, we can thus increase the average intensity of the detected nonlinear signals and increase the acquisition speed and image frame rate (Chu et al., 2003). Previous systems using high repetition-rate $(\sim 80-\mathrm{MHz})$ Ti:sapphire lasers or Er:fiber lasers (Campagnola et al., 1999; Yelin and Silberberg, 1999) for higher harmonic generation microscopy caused either high nonlinear absorption at 800-nm (Chen et al., 2002; König et al., 1997) or linear water absorption at $1550-\mathrm{nm}$, which limits the maximum allowable illumination power and penetration depth with cell vitality penalty. Eight hundred nanometers excitation also faces strong absorption at its THG wavelength ( 266-nm) in live specimens. Other studies using amplifier systems (Müller et al., 1998) were facing the cell viability problem due to extremely high pulse energy (micro-Joule versus pico-Joule from a regular system) while the average signal intensity was limited by the low repetition-rate. In contrast, an all-solid-state Cr:forsterite femtosecond laser (Bouma et al., 1996; Liu et al., 2001; Seas et al., 1992) centered at 1230-nm with a repetition rate of $110 \mathrm{MHz}$ was implemented as the light source for our current study.

\section{Setup of higher harmonics optical microscope}

Fig. 2 shows the experimental setup of the higher harmonic optical microscope (HOM). Our HOM system is adapted from an Olympus FV300 scanning system combined with an Olympus BX51 upright microscope while all optics are modified to allow the passage of 1200-1350-nm infrared (IR) light. A home-built Cr:forsterite laser, which operates at $1230-\mathrm{nm}$ with a repetition rate of $110-\mathrm{MHz}$ and a pulse width of $140-\mathrm{fs}$ (350-mW average output), is used as the light source (Liu et al., 2001) to allow both SHG/THG to fall within the visible spectrum and also provide advantages of low illumination attenuation. The $\mathrm{Cr}$ :forsterite laser constructed for this study uses a 19-mm-long Brewster-cut

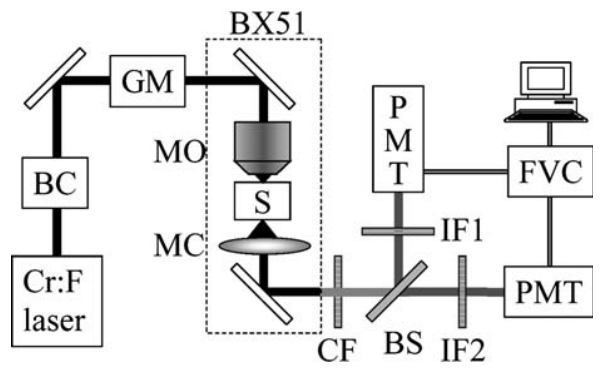

Fig. 2. The schematic of the higher harmonics optical microscope (HOM). BC, beam collimator; GM, galvanometric mirrors; MO, microscope objective; $\mathrm{MC}$, microscope condenser; $\mathrm{CF}$, color filter; $\mathrm{BS}$, beamsplitter; IF, interference filter; PMT, photomultiplier tube; FVC, Fluoview control unit.

Cr:forsterite crystal with its $b$-axis on the horizontal plane, resulting in horizontal polarization for the lasing beam. The crystal temperature is kept around $2{ }^{\circ} \mathrm{C}$. The laser is pumped with $8 \mathrm{~W}$ of $1064 \mathrm{~nm}$ light from a diodepumped Nd: $\mathrm{YVO}_{4}$ laser (Spectra-Physics Millennia IR). The laser cavity is a Z-cavity consisting of mirrors, a $4.5 \%$ output coupler, and a semiconductor saturable absorber mirror (SESAM). A SF6 prism pair is inserted to provide intracavity group-velocity dispersion compensation. All mirrors and the output coupler are highreflection coated for the spectral range of $1200-1270 \mathrm{~nm}$. Before the output coupler, a slit is used to for wavelength tuning. The use of SESAM initializes and stabilizes the Kerr lens mode-locking mechanism in the $\mathrm{Cr}$ :forsterite laser and makes this laser less sensitive to feedback light from the HOM system. On the other hand, the use of SESAM also restricts our tuning range to $1220-1240 \mathrm{~nm}$. Outside the cavity, we use another SF6 prism pair to achieve both beam shaping and dispersion compensation of the laser output. Limited by the spacing between the intracavity prism pair, the repetition rate of the constructed laser is between 60 and $120 \mathrm{MHz}$. For our current study, the central output wavelength is kept at $1230 \mathrm{~nm}$ with a full-width-halfmaximum spectral width of $20 \mathrm{~nm}$ and a repetition rate of $110 \mathrm{MHz}$. The laser output after the external prism pair is shaped and collimated by a telescope and then coupled into the Olympus FV300 scanning system connecting to the Olympus BX51 microscope with an aperture fitting tube lens. Real-time scanning was accomplished through the high-speed galvanometer mirrors inside FV300. The excitation laser pulse is focused into desired locations inside the live specimen and scanned with a spot size close to diffraction limit with a high numerical-aperture (NA) objective. For a complete in vivo imaging of the whole zebrafish embryo that has a diameter around $1.5-\mathrm{mm}$ including the chorion, the working distance of the chosen objective has to be longer than $1.5-\mathrm{mm}$. We choose a $2-\mathrm{mm}$ working distance IR objective (LUMPlanFl/IR 60X/water/NA 0.90, Olympus) to accomplish this goal. The forward-propagating 
optical higher harmonics is collected using a high-NA (1.4) oil-immersion condenser. By using the NA 0.9 long-working-distance objective, the diffraction-limited spatial resolution of our system is $\sim 1-\mu \mathrm{m}$ in axial and 400-nm (500-nm) in radial directions for THG (SHG) microscopy with the $1230-\mathrm{nm}$ excitation wavelength. For HHG intensity mapping, two photomultiplier tubes (R928P, Hamamatsu) are used to record the filtered SHG and THG intensities point by point to form 2D sectioned images. Stereographic 3D images of the whole live specimen can be acquired through accurate height control of the sample cell or objective along the light propagation axis. To observe moving live specimens, the recording speed is chosen to be $0.25-2.5 \mathrm{~s}$ per frame with $512 \times 512$-scanning points.

\section{Developmental dynamics in live zebrafish embryos}

The optical sectioning power and noninvasive nature of SHG and THG allow us to observe the 3D cell proliferation processes in a zebrafish embryo in vivo as shown in Fig. 3. Zebrafish is a modern model vertebrate because of its fecundity, its rapid development, and its scores of available mutants, with easy manipulation of spawning, fertilization, and gene transferring. In addition, zebrafish embryos are transparent, which makes optical observation of embryogenesis possible. In this study, live wild-type zebrafish embryos without removing chorion are kept in a glass aquarium at $28.5^{\circ} \mathrm{C}$ with a $14 \mathrm{~h}$-light $/ 10 \mathrm{~h}$-dark photoperiod. The description of embryonic stages is based on the study of Kimmel et al. (1995).

Due to the optical dispersion property in biological tissues, the contrast in THG microscopy is provided by the inhomogeneity inside a single cell, for instance, the spatial variations of linear or nonlinear susceptibilities between nuclear membranes and cytoplasm or between cell membranes and the surrounding fluid. Moreover, since there is nonvanishing $\chi^{(3)}$ for all materials, THG microscopy can be used as a morphological imaging tool. As a result, the cell structure in the embryo and the distribution of the organelles in the cells can be revealed by in vivo THG microscopy (Fig. 3). On the other hand, SHG images correspond to crystalline nano-structures in biological samples where optical centro-symmetry is broken. Mitotic spindles, for example, are made up of spatially organized dynamic microtubules with a diameter of $\sim 25 \mathrm{~nm}$. Therefore, SHG can be generated from the spindles as the blastoderm cells undergo mitosis (Figs. 3B and $\mathrm{C}$ series). The dynamic changes of spindles and membranes between two daughter cells can be imaged in vivo by the developed HOM system. Endogenous SHG signal vanishes as the spindle microtubules disperse in the cells and exhibit no more crystalline characteristic. No optical damage can be observed during the whole proliferation process after long-term continuous observations even with $100-\mathrm{mW}$ incident average power onto the embryo. Compared with common multi-photon fluorescence technique using 730 800-nm Ti:sapphire femtosecond lasers with a similar experimental condition (König et al., 1997), Chinese hamster ovary cells were found unable to form clones with more than $6-\mathrm{mW}$ average power and complete cell destruction occurs with an average power more than 10$\mathrm{mW}$. Increasing the excitation laser wavelength to $1047 \mathrm{~nm}$ will allow long-term two-photon fluorescence imaging of mammalian embryos without compromising viability with slightly higher excitation power, however the average illumination power was still limited to $13 \mathrm{~mW}$ while a total exposure of only $2 \mathrm{~J}$ could be applied to one embryo within a 24-h imaging period (Squirell et al., 1999). Our successful long-term continuous observation with $100-\mathrm{mW}$ average powers indicates not only that linear absorption (by for example water) induced heating with $1230-\mathrm{nm}$ light is negligible, but also the reduction of multi-photon damages (Chen et al., 2002) with longer wavelength $1230-\mathrm{nm}$ femtosecond light. We can continuously monitor the development of the embryos throughout a 12 -h period of time repeatedly, with a total exposure of over $1000 \mathrm{~J}$ optical energy to one embryo, and the examined embryos can all develop normally to larval stage after they are kept in the container for 1 day.

Besides monitoring the mitotic dynamics at early stage of the embryonic development, the HOM technique also reveals various structures in the fertilized zebrafish eggs, as shown in Fig. 4. In Fig. 4A, THG signals reveal the $0.5-0.7-\mu \mathrm{m}$ width granular canals distributed on the chorion surface, demonstrating the sub-micron resolution of our developed HOM imaging system. Moving 500- $\mu \mathrm{m}$ into the embryo, the distribution of the yolk cells is also outlined by THG emission from the granule membranes (Fig. 4B). Different from the blastomeres, there are neither nucleuses nor organelles inside yolk cells. On the other hand, the semicrystallized membrane proteins in the yolk cells can be picked up by both THG (Fig. 4C) and SHG (Fig. 4D) signals.

Figs. 5 and 6 show the in vivo observation of cell proliferation and embryogenic processes around the animal pole via THG mode at $50 \%$ epiboly stage and shield stage, respectively. The imaging depths for both figures are $\sim 500-\mu \mathrm{m}$ from the chorion surface. These high resolution in vivo images demonstrate the excellent capability of HOM to visualize cellular dynamics deep inside the embryos and this novel noninvasive technique will be very useful in the determination of the cell fate at the early developmental stage.

The excellent depth-resolution and high penetration capability of HOM are also demonstrated in Fig. 7. Individual cell nucleus, cellular membranes, and the 


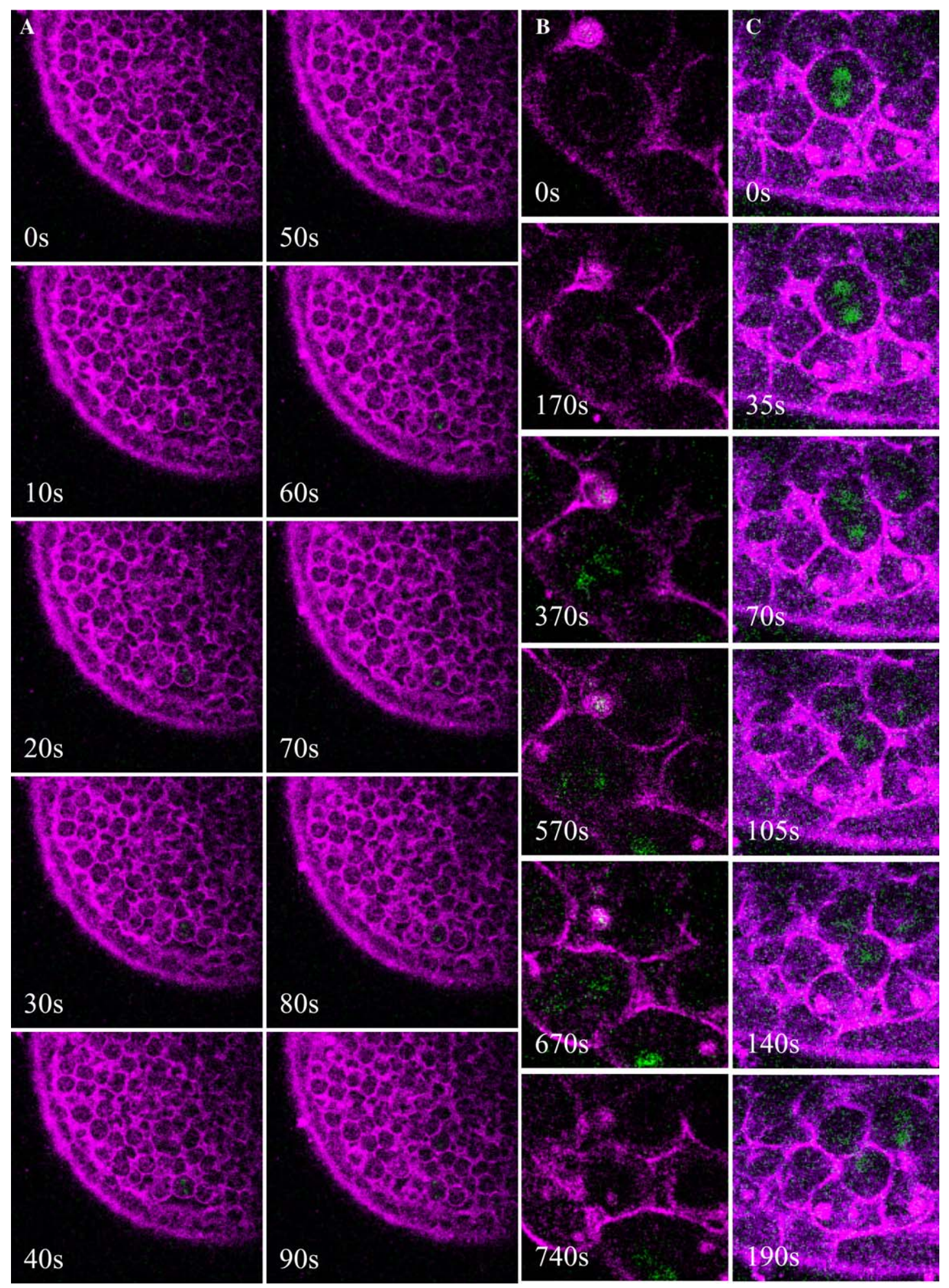

Fig. 3. Mitosis processes inside a live zebrafish embryo in vivo monitored with HOM. (A) A time-series of optical sections inside the embryo at the dome stage. The imaging depth is $\sim 400-\mu \mathrm{m}$ from the chorion surface. THG (shown in purple) picks up all interfaces including external yolk syncytial layers, cell membranes, and nuclear membranes while SHG (shown in green) shows the microtubule-formed spindle biconical arrays. Image size: $235 \times 235-\mu \mathrm{m}^{2}$. (B) A closer view of the mitosis process. The cell in the center is at its transition from interphase to prometaphase $(t=0-170 \mathrm{~s})$ and the dissolution of the nuclear membrane is picked up by the THG modality in vivo. Then the formation and movement of spindles during the anaphase is revealed by the SHG modality $(t=370-570 \mathrm{~s})$. From telophase to cytokinesis, THG shows the elongation of cell and the membrane formation around two daughter cells while SHG diminishes due to the spindle fibers dispersing into cytoplasm. Image size: $60 \times 60-\mu \mathrm{m}^{2}$. (C) Another time series of the mitosis, showing the strong SHG emission from the spindle fiber array. Image size: $60 \times 60-\mu \mathrm{m}^{2}$. 

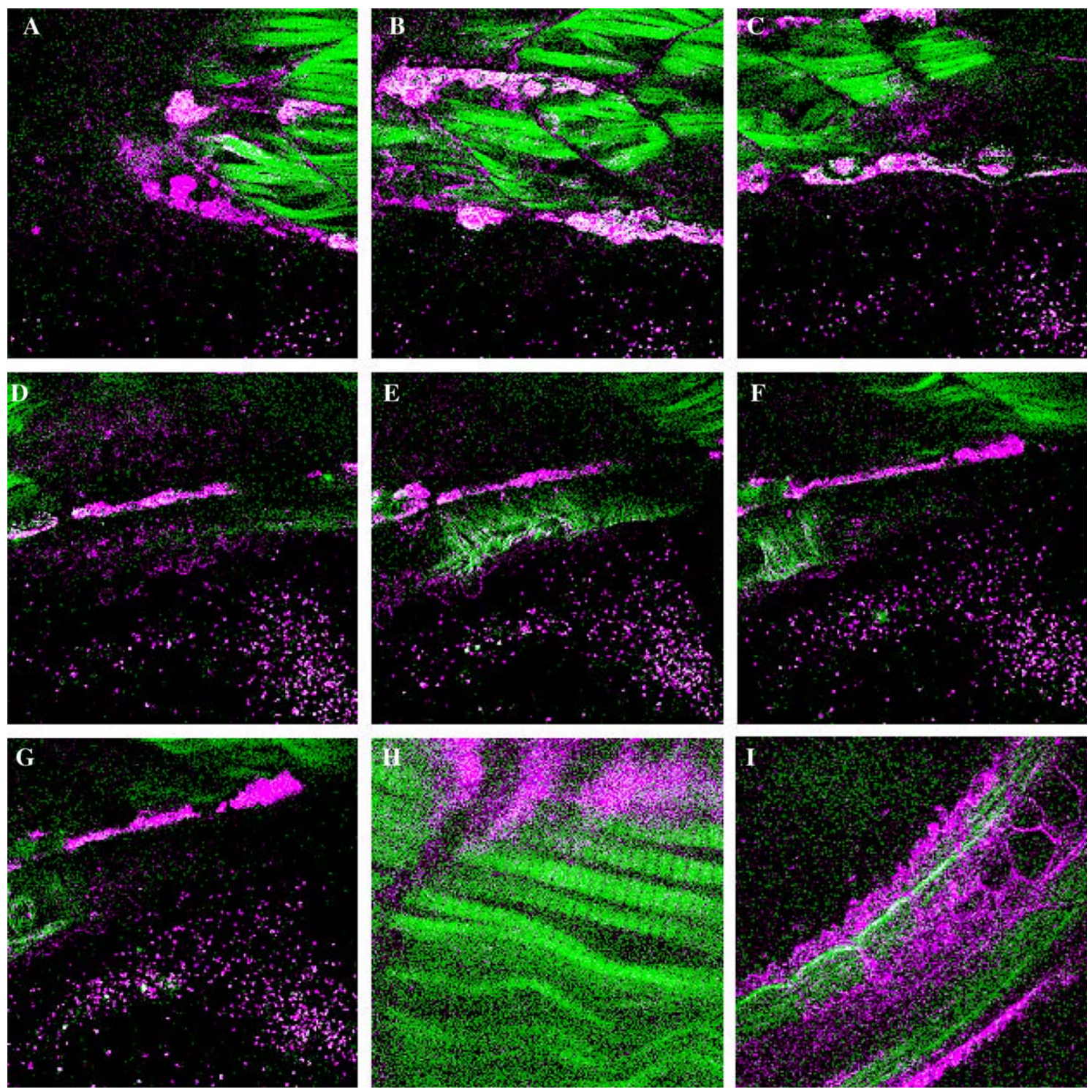

Fig. 10. (A)-(G) An in vivo sectioning series of a swimming zebrafish larva (with dorsad to the bottom of the images) at 5 days after fertilization. (H) The enlarged view inside a somite showing distribution of muscle fibers. (I) An optical section at the center of the larva showing the segments inside the vacuolated notochord and the distribution of somites alongside the notochord. Image size: (A) $-(\mathrm{G})$ and $(\mathrm{I}): 235 \times 235-\mu \mathrm{m}^{2} ;(\mathrm{H}): 40 \times 40-\mu \mathrm{m}^{2}$. THG is shown in purple while SHG is shown in green.

boundary of the blastomere can all be observed through high-resolution THG modality due to their optical susceptibility differences from the surrounding media (Fig. 7A) at a depth of $420-\mu \mathrm{m}$ from the chorion surface. Please notice that due to the cubic dependency on the incident power, THG is more sensitive to the incident power degradation inside the specimen compared with SHG, which has a quadratic dependency on the incident power. Moving 50- $\mu \mathrm{m}$ deeper into the blastoderm (Fig. 7B), the deep cell layers (at left), the yolk cells (at right), and the internal yolk syncytial layers between them all show up in the scanning sectioned THG images, confirming the capability of THG as a general noninvasive imaging tool for cell and tissue morphology. At a depth of $570-\mu \mathrm{m}$ inside the embryo (Fig. 7D), the densely packed yolk granules are clearly observed through THG with excellent spatial resolution, demonstrating its high penetration ability and indicating that no serious aberration is induced for the focused longwavelength IR light even after penetrating $\sim 0.6-\mathrm{mm}$ into the embryo. Currently we can take clear high-resolution (sub-micron) images with a $1.5 \mathrm{~mm}$ penetration through the zebrafish embryo from the chorion surface. Further penetration improvement can be made by taking longer exposure time, by increasing illumination power, or by improving the PMT systems. Fig. 8 shows an in vivo optical sectioning of a whole live zebrafish embryo between 90\%-epiboly and bud stage (left-side view) at a depth of $\sim 600-\mu \mathrm{m}$ from the chorion (chorion is outside the viewing area). This image is composed of 

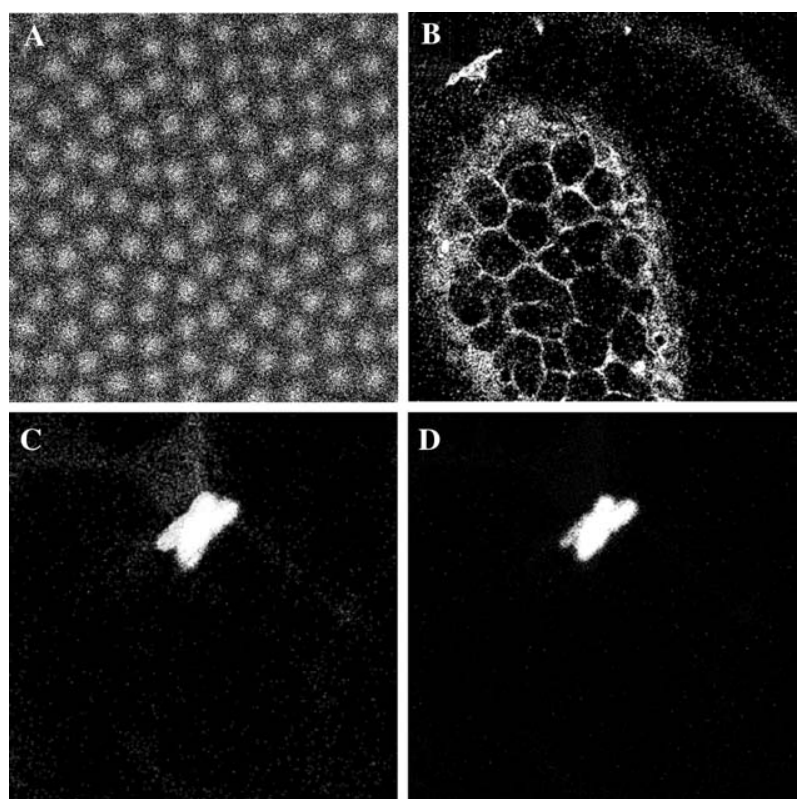

Fig. 4. In vivo optical sections of (A) the chorion surface (THG, $25 \times 25-\mu \mathrm{m}^{2}$ ), (B) the yolk granules (THG, $\left.235 \times 235-\mu \mathrm{m}^{2}\right)$, and (C) THG, (D) SHG: the semi-crystallized membrane protein inside the yolk $\left(50 \times 50-\mu \mathrm{m}^{2}\right)$.

nine individual THG images with a total $2.35 \mathrm{M}$ resolved pixels due to the large scanned area.

Besides observing embryo developments, HOM is also applied to the examination of general tissue/organ structures in live free-swimming larvae. The in vivo THG and SHG optical section series throughout the developing eyes are given in Figs. 9A and B, respectively. The growing retina cells in the eye exhibit strong THG signals due to its higher index. Some gleaming SHG spots can be observed around the edge showing the specific nano-crystalline sites inside the cell. The general morphological structures of larvae, including the epidermis, somite boundary (Figs. 10A-G), and notochord (Fig. 10I), can also be revealed by THG signals. On the other hand, myotomes, which are composed of collaterally organized myosin and actin filaments, exhibit strong SHG emission due to its highly organized nano-structure (Campagnola et al., 2002; Chu et al., 2002), resembling a nonlinear photonic crystal. The myotomes of the larva can be observed in Figs. 10A-C and $\mathrm{E}-\mathrm{G}$. The detailed distribution of muscle fibers in a somite can be revealed (Fig. 10H), where individual sarcomere composed of A- and I-bands with a periodicity less than $2-\mu \mathrm{m}$ can be readily resolved due to the superb spatial resolution provided by this powerful new technique. In Figs. 10E-G, strong SHG emission is also observed in the dorsal fin due to its collagen constituent, resulting in observable SHG. Again this high penetration microscopy allows us to image through a live freeswimming zebrafish larva while sub-micron-scaled morphological developments can be revealed.
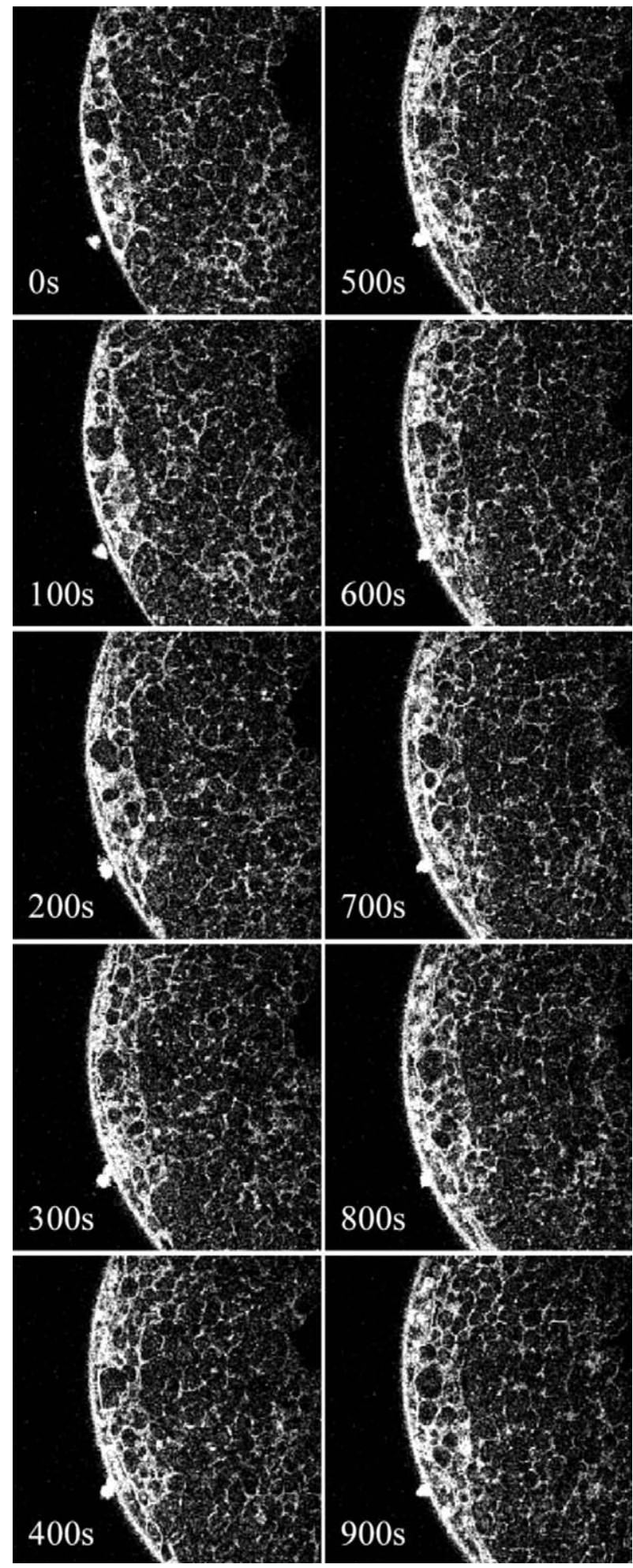

Fig. 5. A THG time series of the in vivo optical sectioning inside the embryo at the $50 \%$ epiboly stage, showing the cell proliferation and epiboly processes with a sub- $\mu \mathrm{m}$ resolution. Image size: $235 \times 235-\mu \mathrm{m}^{2}$.

\section{Conclusion}

The establishment of long-term imaging methods that can maintain the viability of live specimens is critical for 

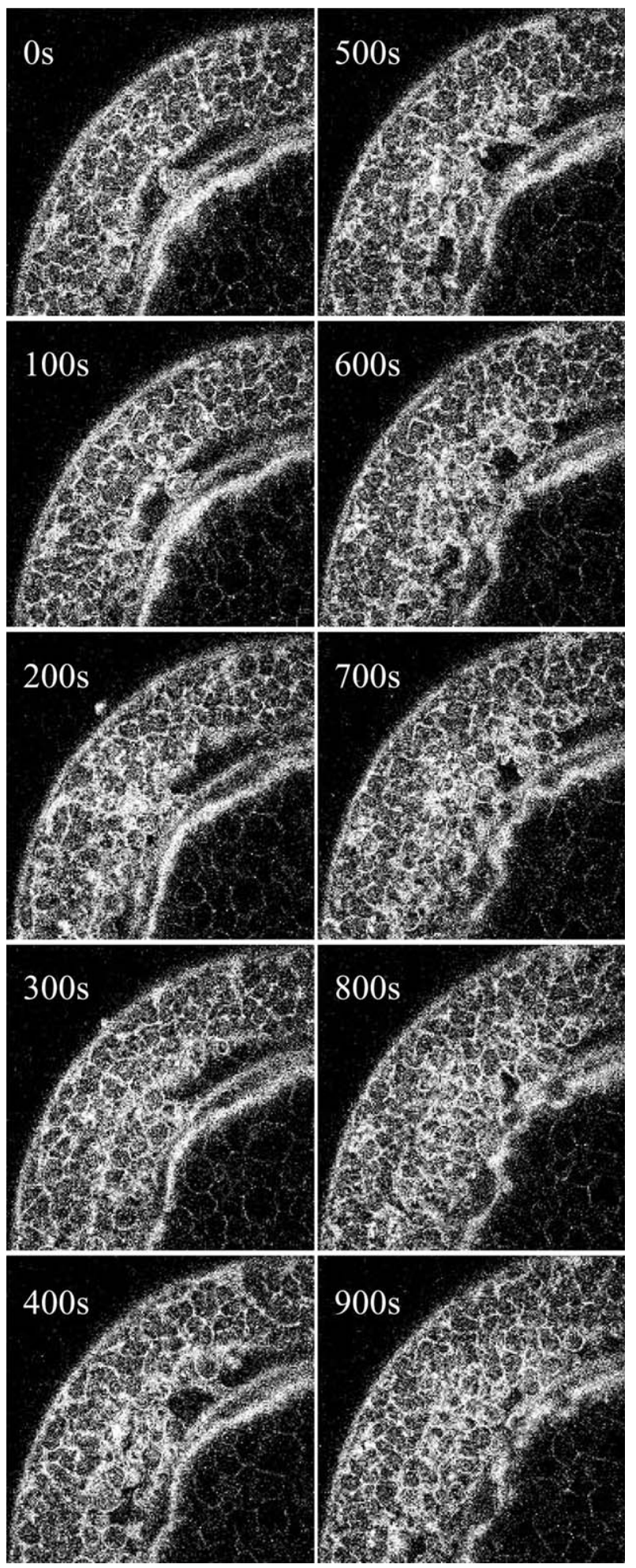

Fig. 6. A THG time series of the in vivo optical sectioning inside the embryo at the shield stage, showing the cell proliferation and epiboly processes with a sub- $\mu \mathrm{m}$ resolution. Image size: $235 \times 235-\mu \mathrm{m}^{2}$.

advancing our knowledge of cell biology and embryonic development. Highly spatially resolved and highly penetrative noninvasive in vivo microscopy has the poten-
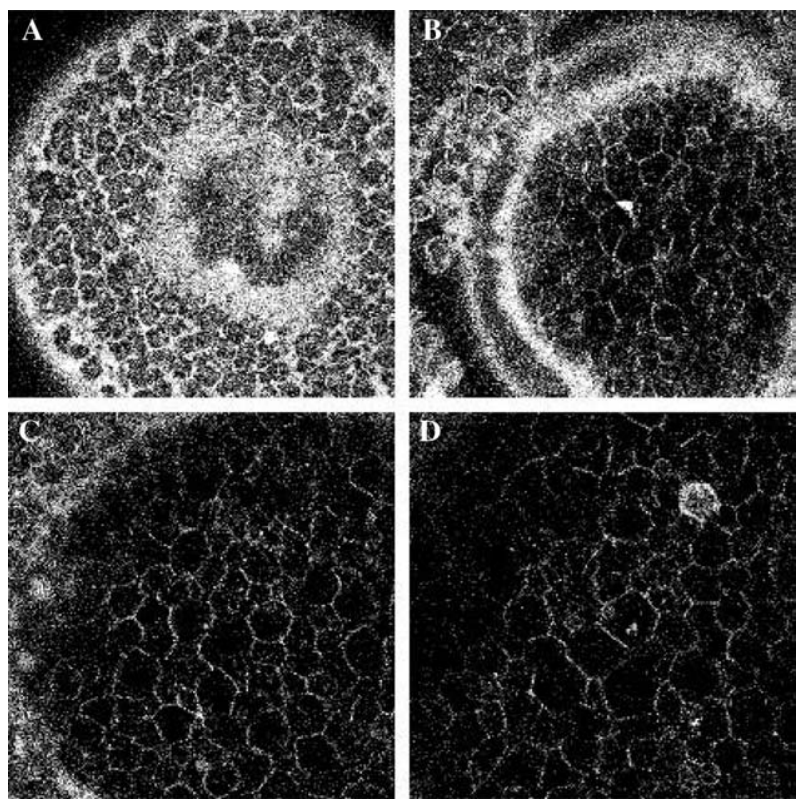

Fig. 7. A depth-resolved THG series sectioned inside the embryo at the $90 \%$ epiboly stage. Depths from chorion for (A)-(D) are 420, 470, 520, and $570-\mu \mathrm{m}$, respectively. Image size: $235 \times 235-\mu \mathrm{m}^{2}$.

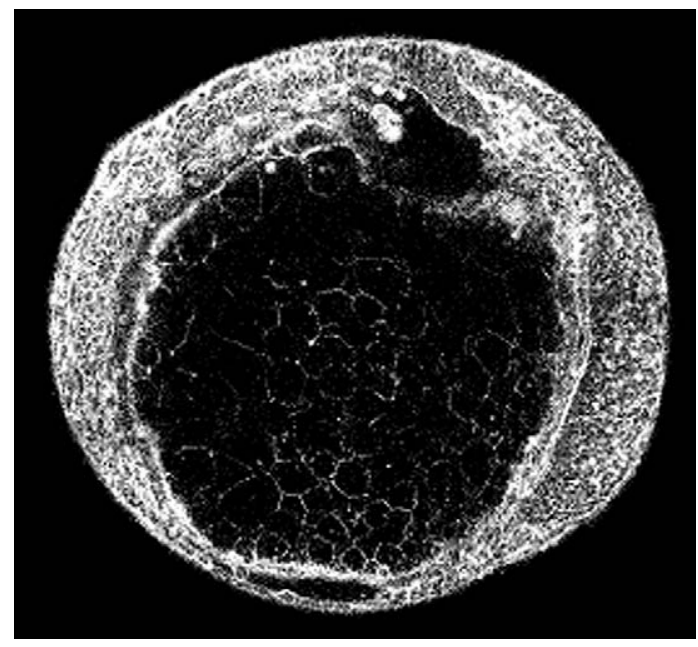

Fig. 8. An in vivo THG optical sectioning (left-side view) of the whole live zebrafish embryo (at the bud stage) at a depth of $\sim 600-\mu \mathrm{m}$ from the chorion surface. Image size: $500 \times 500-\mu \mathrm{m}^{2}$.

tial of offering new insights into the embryonic morphological changes and the complex developmental processes. We have demonstrated that by using endogenous higher harmonic generation as the microscopic contrast mechanism, no fluorescence is required and the common issues of photodamage, phototoxicity, photobleaching, dye availability, or dye toxicity can all be eliminated. By property choosing the excitation source that will allow detectable SHG and THG signals but minimize the unwanted interactions, delicate developmental processes can be in vivo monitored continuously for a long period of time from the initial cell proliferation 


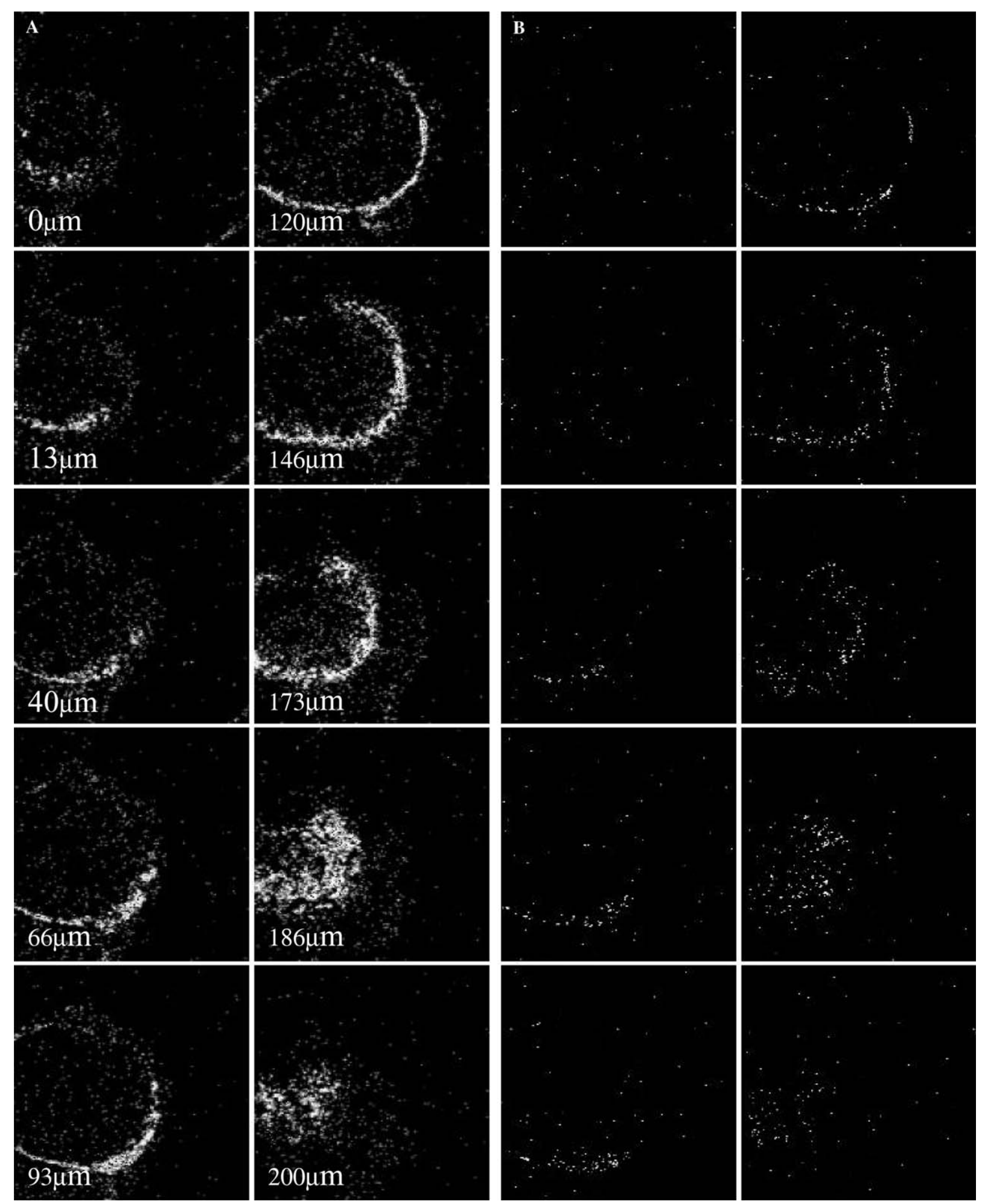

Fig. 9. The in vivo (A) THG and (B) SHG optical section series throughout the developing eye at the prim-12 stage during Pharyngula period. Image size: $235 \times 235-\mu \mathrm{m}^{2}$.

through the formation of tissues inside untreated live vertebrate embryos. Based on a Cr:forsterite femtosecond laser centred at $1230-\mathrm{nm}$, which locates at the transparency window of most biological specimens, we realize a noninvasive and highly penetrative higher- harmonic generation microscopy and the complicated developmental process inside a 1.5 -mm thick zebrafish embryo can be observed clearly in vivo without any treatments on the studied live wild-type specimens. The excellent $3 \mathrm{D}$ resolution $(\sim 0.4-\mu \mathrm{m}$ with $\mathrm{THG})$ of the 
higher-harmonic generation microscopy allows us to capture the subtle developmental processes on the cellular or sub-cellular levels deep inside live embryos. To our knowledge, no other technique can provide such high-resolution, high-contrast in vivo images of developmental processes deep inside live vertebrate embryos and larvae without extra labelling or handling. This demonstrated higher-harmonic generation microscopy, a truly noninvasive microscopy, should be most suitable to the study of the 3D cytoarchitecture dynamics during embryogenesis with sub-micrometer resolution and millimeter penetration depth. In addition, this approach may be potentially useful for studying cell-fate determination at early developmental stages.

\section{Acknowledgments}

The authors would like to acknowledge the support by the National Health Research Institute (NHRI-EX929201EI), National Science Council (NSC-91-2215-E-002021), and NTU Center for Genomic Medicine of Taiwan, ROC. S.W. Chu would like to acknowledge the generous support from MediaTek Incorporation.

\section{References}

Anderson, R.R., Parish, J.A., 1981. The optics of human skin. J. Invest. Dermat. 77, 13-19.

Barad, Y., Eisenberg, E., Horowitz, M., Silberberg, Y., 1997. Nonlinear scanning laser microscopy by third harmonic generation. Appl. Phys. Lett. 70, 922-924.

Battaglia, A., 2003. Neuroimaging studies in the evaluation of developmental delay/mental retardation. Am. J. Med. Genet. C 117, 25-30.

Bouma, B.E., Tearney, G.J., Bilinsky, I.P., Golubovic, B., Fujimoto, J.G., 1996. Self-phase-modulated Kerr-lens mode-locked Cr:forsterite laser source for optical coherence tomography. Opt. Lett. 21, 1839-1841.

Boppart, S.A., Tearney, G.J., Bouma, B.E., Southern, J.F., Brezinski, M.E., Fujimoto, J.G., 1997. Noninvasive assessment of the developing Xenopus cardiovascular system using optical coherence tomography. Proc. Natl. Acad. Sci. 94, 4256-4261.

Brown, E., McKee, T., diTomaso, E., Pluen, A., Seed, B., Boucher, Y., Jain, R.K., 2003. Dynamic imaging of collagen and its modulation in tumors in vivo using second-harmonic generation. Nat. Med. 9, 796-800.

Campagnola, P.J., Wei, M.D., Lewis, A., Loew, L.M., 1999. High resolution nonlinear optical imaging of live cells by second harmonic generation. Biophys. J. 77, 3341-3349.

Campagnola, P.J., Millard, A.C., Terasaki, M., Hoppe, P.E., Malone, C.J., Mohler, W.A., 2002. Three-dimensional high-resolution second-harmonic generation imaging of endogenous structural proteins in biological tissues. Biophys. J. 82, 493-508.

Chen, I.-H., Chu, S.-W., Sun, C.-K., Lin, B.-L., Cheng, P.-C., 2002. Wavelength dependent damage in biological multi-photon confocal microscopy: a micro-spectroscopic comparison between femtosecond Ti:sapphire and Cr:forsterite laser sources. Opt. Quantum. Electron 34, 1251-1266.
Chu, S.W., Chen, I.-H., Liu, T.-M., Cheng, P.-C., Sun, C.-K., Lin, B.L., 2001. Multimodal nonlinear spectral microscopy based on a femtosecond Cr:forsterite laser. Opt. Lett. 26, 1909-1911.

Chu, S.W., Chen, I.-H., Liu, T.-M., Sun, C.-K., Lee, S.-P., Lin, B.-L., Cheng, P.-C., Kuo, M.-X., Lin, D.-J., Liu, H.-L., 2002. Nonlinear bio-photonic crystal effects revealed with multi-modal nonlinear microscopy. J. Microsc. 208, 190-200.

Chu, S.-W., Liu, T.-M., Sun, C.-K., Lin, C.-Y., Tsai, H.-J., 2003. RealTime Second-Harmonic-Generation Microscopy Based on a 2GHz Repetition Rate Ti:sapphire Laser. Opt. Express 11, 933938

Drexler, W., Morgner, U., Ghanta, R.K., Kärtner, F.X., Schuman, J.S., Fujimoto, J.G., 2001. Ultrahigh-resolution ophthalmic optical coherence tomography. Nat. Med. 7, 502-507.

Denk, W., Strickler, J.H., Webb, W.W., 1990. Two-photon laser scanning fluorescence microscopy. Science 248, 73-76.

Freund, I., Deutsch, M., Sprecher, A., 1986. Connective Tissue Polarity: Optical Second-harmonic Microscopy, Crossed-beam Summation, and Small-angle Scattering in Rat-tail Tendon. Biophys. J. 50, 693-712.

Gannaway, J.N., Sheppard, C.J.R., 1978. Second-harmonic imaging in the scanning optical microscope. Opt. Quantum Electron. 10, 435439.

Greicius, M.D., 2003. Neuroimaging in developmental disorders. Curr. Opin. Neurol. 16, 143-146.

Guo, Y., Ho, P.P., Tirksliunas, A., Liu, F., Alfano, R.R., 1997. Optical harmonic generation from animal tissues by the use of picosecond and femtosecond laser pulses. Opt. Lett. 22, 1323-1325.

Kimmel, C.B., Ballard, W.W., Kimmel, S.R., Ullmann, B., Schilling, T.F., 1995. Stages of embryonic development of the zebrafish. Dev. Dyn. 203, 253-310.

König, K., So, P.T.C., Mantulin, W.W., Gratton, E., 1997. Cellular response to near-infrared femtosecond laser pulses in two-photon microscopes. Opt. Lett. 22, 135-136.

Liu, T.-M., Chu, S.-W., Sun, C.-K., Lin, B.-L., Cheng, P.C., Johnson, I., 2001. Multi-photon scanning microscopy using a femtosecond Cr:forsterite laser. Scanning 23, 249-254.

Moreaux, L., Sandre, O., Blanchard-Desce, M., Mertz, J., 2000. Membrane imaging by simultaneous second-harmonic generation and two-photon microscopy. Opt. Lett. 25, 320-322.

Müller, M., Squier, J., Wilson, K.R., Brakenhoff, G.J., 1998. 3D microscopy of transparent objects using third-harmonic generation. J. Microsc. 191, 266-274.

Palmes-Saloma, C., Saloma, C., 2000. Long-depth imaging of specific gene expressions in whole-mount mouse embryos with singlephoton excitation confocal fluorescence microscopy and FISH. J. Struct. Biol. 131, 56-66.

Seas, A., Petričević, V., Alfano, R.R., 1992. Generation of sub-100-fs pulses from a $\mathrm{CW}$ mode-locked chromium-doped forsterite laser. Opt. Lett. 17, 937-939.

Peleg, G., Lewis, A., Linial, M., Loew, L.M., 1999. Nonlinear optical measurement of membrane potential around single molecules at selected cellular sites. Proc. Natl. Acad. Sci. 96, 6700-6704.

Phillips, C.L., Arend, L.J., Filson, A.J., Kojetin, D.J., Clendenon, J.L., Fang, S., Dunn, K.W., 2001. Three-dimensional imaging of embryonic mouse kidney by two-photon microscopy. Am. J. Pathol. 158, 49-55.

Povazay, B., Bizheva, K., Unterhuber, A., Hermann, B., Sattmann, H., Fercher, A.F., Drexler, W., Apolonski, A., Wadsworth, W.J., Knight, J.C., Russell, P., St, J., Vetterlein, M., Scherzer, E., 2002. Submicrometer axial resolution optical coherence tomography. Opt. Lett. 27, 1800-1802.

Shen, Y.R., 1989. Surface properties probed by 2 nd harmonic and sum frequency generation. Nature 337, 519-525.

Squirell, J.M., Wokosin, D.L., White, J.G., Bavister, B.D., 1999. Long-term two-photon imaging of mammalian embryos without compromising viability. Nat. Biotechnol. 17, 763-767. 
Stelzer, E.H.K., Hell, S., Lindek, S., Stricker, R., Pick, R., Storz, C., Ritter, G., Salmon, N., 1994. Nonlinear absorption extends confocal fluorescence microscopy into the ultra-violet regime and confines the illumination volume. Opt. Commun. 104, 223228.

Sun, C.-K., Chu, S.-W., Tai, S.-P., Keller, S., Mishra, U.K., DenBaars, S.P., 2000. Scanning second-harmonic-generation and third-harmonic-generation microscopy of GaN. Appl. Phys. Lett. 77, 23312333.

Sun, C.-K., Chu, S.W., Tai, S.P., Keller, S., Abare, A., Mishira, U.K., DenBaars, S.P., 2001. Mapping piezoelectric-field distribution in gallium nitride with scanning second-harmonic generation microscopy. Scanning 23, 182-192.
Turnbull, D.H., Bloomfield, T.S., Baldwin, H.S., Foster, F.S., Joyner, A.L., 1995. Ultrasound backscatter microscope analysis of early mouse embryonic brain development. Proc. Natl. Acad. Sci. 92, 2239-2243.

Yeh, A.T., Nassif, N., Zoumi, A., Tromberg, B., 2002. Selective corneal imaging using combined second-harmonic generation and two-photon excited fluorescence. Opt. Lett. 27, 2082-2084.

Yelin, D., Silberberg, Y., 1999. Laser scanning third-harmonicgeneration microscopy in biology. Opt. Express 5, 169-175.

Zoumi, A., Yeh, A., Tromberg, B., 2002. Imaging cells and extracellular matrix in vivo by using second harmonic generation and twophoton excited fluorescence. Proc. Natl. Acad. Sci. 99, 11014 11019 . 